\title{
Um modelo iterativo para solução de cabos pré-tracionados sob ação de cargas uniformemente distribuídas
}

\author{
Covre, L. ${ }^{*} ;$ Loeffler, C. F. ${ }^{1,2}$ \\ 1 Departamento de Engenharia Mecânica, Universidade Federal do Espírito Santo, Vitória, ES, Brasil. \\ 2 Programa de Pós-Graduação em Engenharia Mecânica, Universidade Federal do Espírito Santo, Vitória, ES, Brasil. \\ *e-mail:lucas_loriato@hotmail.com
}

\begin{abstract}
Resumo
Este trabalho apresenta um modelo simplificado não linear do comportamento mecânico de cabos tracionados, sujeito a carregamentos transversais. Existem diversos modelos para tais estruturas flexíveis, que se distinguem pela aplicação de hipóteses simplificadoras, principalmente quanto ao formato da curva, a consideração de rotações pequenas ou moderadas, o modo de distribuição do carregamento e a variação da tração ao longo do cabo, entre outras. Este estudo utiliza dois modelos simples, mas não lineares. Um deles parte do modelo simplificado comumente chamado de teoria das cordas, que adota uma aproximação parabólica para o formato da curva; o outro assume a forma catenária do cabo. Em ambos, as rotações no cabo interagem com as deformações lineares axiais, gerando um esquema iterativo em que a rigidez é atualizada em função dos deslocamentos transversais do cabo. Os modelos são comparados entre si, através da solução de um mesmo exemplo.
\end{abstract}

\begin{abstract}
This paper presents a simplified nonlinear model of the mechanical behavior of traction cables, subject to cross loads. There are several models for such flexible structures, which are distinguished by the application of simplifying hypotheses, mainly regarding the shape of the curve, the consideration of small or moderate rotations, the mode of distribution of the load and the variation of the traction along the cable, among others. This study uses two simple but non-linear models. One of them is part of the simplified model commonly called string theory, which adopts a parabolic approximation to the shape of the curve; the other takes the catenary form of the cable. In both, rotations in the cableinteract with the axial linear deformations, generating an iterative scheme in which the stiffness is updated as a function of the transverse displacements of the cable. The models are compared to each other, through the solution of the same example.
\end{abstract}

Keywords: Theory of Cables; small strain rotation; Tensor problems;

\section{Introdução}

Os cabos são elementos estruturais flexíveis, longos e delgados, projetados para suportar cargas transversais através da alteração da sua conformação geométrica original. Tal alteração gera esforços axiais internos em que compensa as cargas externas aplicadas.

$\mathrm{O}$ uso industrial dos cabos é imenso. Estruturalmente podem ser citadas as aplicações em pontes, teleféricos, linhas de transmissão e torres estaiadas [1]. 
Duas observações importantes devem ser enunciadas para a melhor abordagem do tema. A primeira ressalta que este trabalho aborda estruturas delgadas flexíveis.

Os livros de mecânica básica e cálculo diferencial abordam esforços e deslocamentos transversais em cabos, mas considerando-os inextensíveis. Com tal hipótese avalia-se o efeito do peso próprio na determinação do formato da curva - uma catenária cuja particularização depende do tamanho do vão, que necessariamente é menor do que o seu comprimento original. Matematicamente, a solução deste problema é um episódio histórico, empreendido pelos irmãos Bernoulli no século XV [2,3.].De fato, as equações empregadas no equilíbrio da corda inextensível podem ser usadasna descrição do comportamento preliminar dos cabos, particularmente no que tange a estabilidade dos esforços; mas, a semelhança se esgota rapidamente, uma vez que os cabos são flexíveis e o modo de deformação é fundamental para sua descrição estrutural [4].

A segunda observação consiste na distinção entre a teoria das cordas flexíveis em que se busca determinar as flechas segundo um modelo parabólico e linear [5]. Nestes considera-se o equilíbrio de forças uma fatia elementar da corda posicionada numa configuração deformada, mas não se faz uma atualização do valor da tração na corda, que rigorosamente é uma função dos deslocamentos. Não se trata, portanto, de uma análise estrutural, que demanda uma correlação entre as rotações na estrutura [6] e o acréscimo de esforços. Por fim, uma vez que não há unanimidade quanto à nomenclatura, neste trabalho a denominação de cabo caracteriza aqui qualquer elemento estrutural flexível que,sob ação de cargas transversais, se equilibre gerando esforços axiais. Os deslocamentos transversais garantem a necessária rigidez. Inclui-se aqui, portanto, as cordas e as estruturas conhecidas como tirantes.

\section{Teoria Geral dos Cabos}

Um cabo é um membro delgado e flexível capaz de desenvolver apenas tensões trativas distribuídas uniformemente na seção, poisque praticamente não possui rigidez flexional [7]. O cabo resiste às cargas transversais por meio de mudanças significativas na inclinação do mesmo nos pontos de aplicação da carga, ou seja, experimenta rotações significativas. Isso faz com que componentes de força sejam desenvolvidas de forma transversal ao comprimento ao longo do cabo, estabelecendo-se assim o necessário equilíbrio. É conveniente então determinar o formato que 0 cabo desenvolve nas condições de carregamento.

Dessa forma, considera-se o cabo mostrado na Figura 1 suspenso entre dois pontos $A$ e $B$ e sujeito a um sistema generalizado de cargas aplicadas $p(x)$.

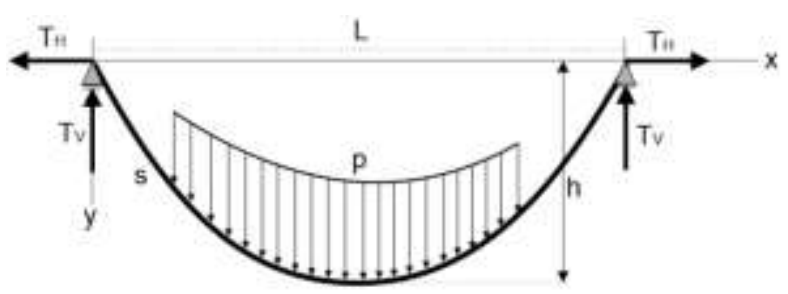

Figura 1- Geometria e estática de um cabo simples.

A linha horizontal é o chamado vão, de comprimento $L$. No estudo de cabos inextensíveis, o vão é sempre menor do que o comprimento do cabo $S$. Já no estudo de cabos extensíveis, o comprimento original do cabo também é $L$; apenas com a deformação seu comprimento se altera. Particularmente nisto está o primeiro importante distintivo no estudo de cabos extensíveis e inextensíveis. O mais importante, contudo, se refere ao fato de que a deformação do cabo altera o valor da sua tração, o que conduz à não linearidade do modelo. Outras condições, referentes àcinemática do modelo também são distintas. Contudo, as equações de equilíbriosão similares, o que muitas vezes cria certa confusão.

O cabo adquire uma determinada forma, $w=w(x)$, que promove equilíbrio.Considerando-se que nenhuma força horizontal age na estrutura, a componente horizontal de tensão no cabo $T_{H}$ se auto equilibra em cada segmento do cabo. Contudo, este valor não é uma constante, alterando-se com a deflexão do cabo.

Partindo da estática do segmento do cabo mostrada na Figura 2, vê-se que a tração $T$ em qualquer ponto do cabo se relaciona com a componente $T_{H}$ através da seguinte expressão: 
Assim, uma vez que o braço de alavanca é igual

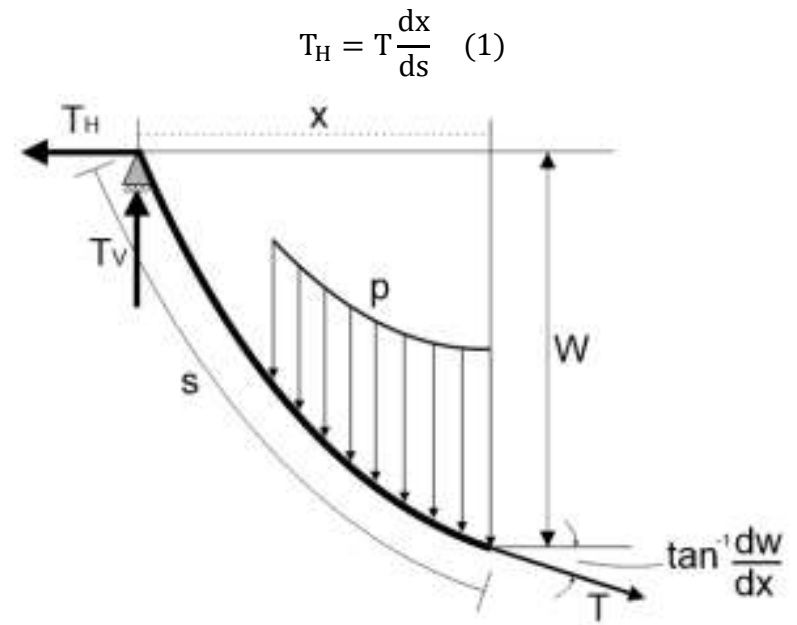

Figura 2- Geometria e estática de um cabo simples.

Na última equação, s é o comprimento medido ao longo do eixo deformado do cabo. Como, por definição, a rigidez à flexão do cabo é nula, o momento fletor emqualquer seção é considerada desprezível. Assim, uma vez que se determine a reação vertical nas extremidades de amarração do cabo, que serão iguais se o carregamento no cabo for uniformemente distribuído, pode-se escrever que:

$$
\mathrm{T}_{\mathrm{H}} \mathrm{w}(\mathrm{x})+\mathrm{M}_{\mathrm{P}}(\mathrm{X})-\mathrm{T}_{\mathrm{V}} \mathrm{x}=0 \text { (2) }
$$

$\mathrm{M}_{\mathrm{P}}(\mathrm{X})$ é o momento produzido pelo carregamento atuante no cabo. Se for o peso próprio, é precisocomputar o comprimento $s$ do cabo para gerar uma carga por unidade de comprimento. Conforme exposto, $T_{H}$ não é constante, mas será admitido como tal num primeiro momento, em que se define preliminarmente o modelo cinemático do cabo.

\section{Aproximação parabólica}

O caso de um cabo uniformemente carregado é um exemplo clássico. Neste caso, as reações em cada apoio são iguais, de valor correspondente a carga aplicada por unidade de comprimento $p$ versus o comprimento do vão $L$ :

$$
\mathrm{T}_{\mathrm{V}}=\frac{\mathrm{pL}}{2}
$$
àdistância $x$ entre o ponto considerado e os apoios, o momento da força $M_{p}(X)$ é dado por:

$$
\mathrm{M}_{\mathrm{P}}(\mathrm{X})=\frac{\mathrm{px}^{2}}{2}
$$

Observe-se que rigorosamente o momento $M_{p}(X)$ seria o produto p.x versus metade do comprimento s. Com tal simplificação, tomando $s \approx x$, da equação (2) resulta:

$$
\mathrm{T}_{\mathrm{H}} \mathrm{W}(\mathrm{x})=\frac{\mathrm{px}}{2}(\mathrm{~L}-\mathrm{x})
$$

Dessa forma, o cabo adquire o formato de uma parábola. Isolando $w(x)$, tem-se:

$$
\mathrm{w}(\mathrm{x})=\frac{\mathrm{p} x}{2 \mathrm{~T}_{\mathrm{H}}}(\mathrm{L}-\mathrm{x})
$$

Denotando o arqueamento do cabo em $x=L / 2$ por $h$, obtém-se, a partir da equação (5):

$$
\mathrm{T}_{\mathrm{H}}=\frac{p \mathrm{~L}^{2}}{8 \mathrm{~h}}
$$

Consequentemente, substituindo (7) em (6), tem-se:

$$
w(x)=\frac{4 h x}{L^{2}}(L-x)
$$

A derivada de $w(x)$ pode ser facilmente calculada:

$$
\frac{\mathrm{dw}(\mathrm{x})}{\mathrm{dx}}=\frac{4 \mathrm{~h}}{\mathrm{~L}}-\frac{2 \mathrm{hx}}{\mathrm{L}^{2}}=\frac{4 \mathrm{~h}}{\mathrm{~L}}\left(1-\frac{\mathrm{x}}{2 \mathrm{~L}}\right)
$$

A tensão total no cabo pode ser agora determinada. Da equação (1), percebe-se que:

$$
\mathrm{T}=\mathrm{T}_{\mathrm{H}} \frac{\mathrm{ds}}{\mathrm{dx}}=\mathrm{T}_{\mathrm{H}}\left[1+\left(\frac{\mathrm{dw}}{\mathrm{dx}}\right)^{2}\right]^{\frac{1}{2}}
$$

Assim, substituindo (9) em (10), tem-se:

$$
\mathrm{T}=\mathrm{T}_{\mathrm{H}}\left[1+16 \mathrm{Y}^{2}\left(1-\frac{2 \mathrm{x}}{\mathrm{L}}\right)^{2}\right]^{\frac{1}{2}}
$$




\section{Encontro Científico de Física Aplicada}

Na expressão anterior, $\mathrm{Y}=\mathrm{h} / \mathrm{L}$ é chamado de razão de arqueamento do cabo. Percebe-se que a máxima tensão ocorre em nos pontos de fixação do cabo, onde a inclinação do cabo é maior, com magnitude igual a:

$$
\mathrm{T}_{\mathrm{MAX}}=\mathrm{T}_{\mathrm{H}}\left[1+4 \mathrm{\gamma}^{2}\right]^{\frac{1}{2}}
$$

A tensão no cabo em qualquer ponto é obtida dividindose a força no cabo pela área da seção reta resistente. Utilizando a equação (10), tem-se:

$$
\sigma(x)=\sigma_{H}\left[1+\left(\frac{d w}{d x}\right)^{2}\right]^{\frac{1}{2}}=\sigma_{H}\left[1+\left(\frac{d w}{d x}\right)^{2}\right]^{\frac{1}{2}}
$$

O termo em colchetes corresponde à forma simplificada do tensor de Euler-Lagrange [8], aplicável aos problemas caracterizados por pequenas rotações.

$$
\varepsilon(\mathrm{x})=\left[1+\left(\frac{\mathrm{dw}}{\mathrm{dx}}\right)^{2}\right]^{\frac{1}{2}}
$$

\section{Aproximação hiperbólica}

Nesta teoria, como em qualquer outrareferente aos cabos, a rigidez de flexão do cabo é considerada desprezível e o momento fletor em qualquer seção é nulo. Também a componente vertical da reação nos apoios se deve diretamente ao carregamento aplicado. Isto fica demonstrado quandose faz o somatório das forças no ponto de inflexão do cabo, em que a tensão no cabo é dada apenas pela componente horizontal.Nestas condições, considerandoque 0 carregamento no cabo seja uniformemente distribuído, tem-se as seguintes equações de equilíbrio horizontal e vertical:

$$
\mathrm{T}_{\mathrm{H}}=\mathrm{T}_{0}(15 \cdot a) \mathrm{T}_{\mathrm{V}}=\mathrm{ps}
$$

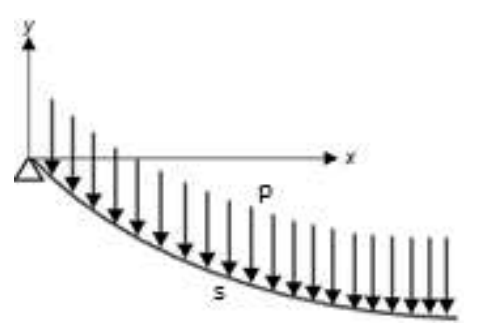

A aproximação hiperbólica se distingue da parabólica neste ponto: agora o momento da força $M_{p}(X)$ (vide eq. 4) é dado de modo mais realístico, considerando o comprimento do cabo:

$$
\mathrm{M}_{\mathrm{P}}(\mathrm{X})=\frac{\mathrm{pxs}}{2}
$$

Esta teoria adota-se um caminho estratégico para seu desenvolvimento. Sabe-se que a relação entre $T_{v}$ e $T_{h}$ em qualquer ponto da corda é:

$$
\frac{\mathrm{T}_{\mathrm{v}}}{\mathrm{T}_{\mathrm{o}}}=\tan \theta=\frac{\mathrm{dW}}{\mathrm{dx}}
$$

Substituindo (15.b) em(16), obtém-se:

$$
\frac{\mathrm{dW}}{\mathrm{dx}}=\frac{\mathrm{ps}}{\mathrm{T}_{\mathrm{o}}}
$$

Como $s$ é uma função de $x$, ou seja, $s=s(x)$, a mesma pode ser derivada em relação a $x$. Assim, derivando a equação (17):

$$
\frac{\mathrm{d}^{2} \mathrm{~W}}{\mathrm{dx}^{2}}=\frac{\mathrm{d}}{\mathrm{dx}} \frac{p}{\mathrm{~T}_{\mathrm{o}}} \mathrm{s}(\mathrm{x})=\frac{\mathrm{p}}{\mathrm{T}_{\mathrm{o}}} \frac{\mathrm{ds}}{\mathrm{dx}}
$$

O infinitesimal $d s$, onde $s$ é o comprimento da corda, é dado por:

$$
d s^{2}=d x^{2}+d W^{2}
$$

Assim, dividindo-se ambos os lados por $d x^{2}$ :

$$
\frac{\mathrm{ds}^{2}}{d x^{2}}=\frac{d x^{2}}{d x^{2}}+\frac{d W^{2}}{d x^{2}} \rightarrow \frac{d s}{d x}=\sqrt{1+\left(\frac{d W}{d x}\right)^{2}}
$$

Substituindo a relação (20) na equação (18), obtém-se:

$$
\frac{\mathrm{d}^{2} \mathrm{~W}}{\mathrm{dx}^{2}}=\frac{p}{\mathrm{~T}_{\mathrm{o}}} \sqrt{1+\left(\frac{\mathrm{dW}}{\mathrm{dx}}\right)^{2}}
$$

É comum se encontrar uma forma aproximada para a representação do radical existente na expressão (21) em problemas em que o modelo parabólico é utilizado. Neste caso, a expressão anterior fica dada por: 


$$
\frac{\mathrm{d}^{2} \mathrm{~W}}{\mathrm{dx}^{2}}=\frac{p}{\mathrm{~T}_{\mathrm{o}}}\left[1+\frac{1}{2}\left(\frac{\mathrm{dW}}{\mathrm{dx}}\right)\right]=\mathrm{c}\left[1+\frac{1}{2}\left(\frac{\mathrm{dW}}{\mathrm{dx}}\right)\right]
$$

Curiosamente, a adoção dessa hipótese simplificadora, efetiva na aproximação parabólica dos cabos, resulta numa solução muito complicada, que torna a realização do posterior processo iterativo inviável. Dessa forma, resolvendo o problema conforme dado pela equação (21), tem-se:

$$
\mathrm{W}(\mathrm{x})=\frac{\sinh \left(\mathrm{k}_{1}\right) \sinh (\mathrm{cx})+\cosh \left(\mathrm{k}_{1}\right) \cosh (\mathrm{cx})}{\mathrm{c}}+\mathrm{k}_{2}
$$

Utilizando as condições de contorno:

$$
\mathrm{W}(0)=0 \quad(24 . \mathrm{a}) \mathrm{W}^{\prime}\left(\frac{L}{2}\right)=0
$$

Nas equações anteriores, Lé o comprimento inicial do cabo. Assim, encontram-se as constantes $k_{1}$ e $k_{2}$ :

$$
\mathrm{k}_{1}=-\frac{\mathrm{cL}}{2}(25 . \mathrm{a}) \mathrm{k}_{2}=-\frac{\cosh (-0,5 \mathrm{cL})}{\mathrm{c}}
$$

Assim, substituindo (25.a) e (25.b) em (23),

$$
\begin{gathered}
\mathrm{W}(\mathrm{x})=\frac{\sinh (-0,5 \mathrm{c} L) * \sinh (\mathrm{cx})}{\mathrm{c}}+ \\
+\frac{\cosh (-0,5 \mathrm{cL}) * \cosh (\mathrm{cx})-\cosh (-0,5 \mathrm{cL})}{\mathrm{c}}(26)
\end{gathered}
$$

\section{Procedimento Iterativo de Solução}

O deslocamento transversal do cabo altera a rigidez do mesmo, de forma que o problema é não linear e somente pode ser resolvido iterativamente. Como em qualquer procedimento iterativo, a primeira solução se baseia num modelo linear em que uma estimativa é feita com relação a algum parâmetro que por sua vez é função de outra variável. No caso, adota-se um valor da tração inicial aplicada ao cabo durante a montagem. Sabe-se que pela ação do carregamento e seu valor se amplia; contudo, admite-se esse valor como constante, determinando a curva inicial de deslocamentos transversais. Essa curva pode obedecer ao modelo parabólico ou ao hiperbólico.
Definida a expressão dos deslocamentos, determina-se o novo comprimento do cabo e assim atualiza-se o valor da tração no cabo. Passa-se ao cálculo no seu novo comprimento, feito a partir da seguinte relação:

$$
\mathrm{L}_{1}=2 \int_{0}^{\frac{L}{2}}\left[1+\left(\frac{\mathrm{dW}}{\mathrm{dx}}\right)^{2}\right]^{\frac{1}{2}} \mathrm{dx}
$$

O valor de $W$ depende do tipo de aproximação escolhido. É possível realizar-se um processo iterativo constituído de várias iterações, de forma que sempre se irá calcular um novo comprimento total deformado e o alongamento máximo na direção $y$ atualizando o valor da carga $T$ aplicada e, consequentemente o valor de $c$. Pode-se escrever o passo a passo iterativo para ambos os tipos de aproximações de uma forma genérica,atribuindo-se índices $i$ às equações. Assim:

$$
c_{i}=\frac{p}{T_{i}}
$$

Para ambos os tipos de aproximação, resolvendo a integral em (27), obtém-se funções que variam com $c$ :

$$
\mathrm{L}_{\mathrm{i}+1}=\mathrm{f}\left(c_{i}\right)
$$

O acréscimo de carga na direção horizontal devido à deformação do cabo pode ser escrito como:

$$
\mathrm{T}^{\prime}{ }_{\mathrm{i}+1}=\frac{\mathrm{E} * \Delta \mathrm{L}_{\mathrm{i}+1} * \mathrm{~A}}{L}
$$

Onde:

$$
\Delta \mathrm{L}_{\mathrm{i}+1}=\mathrm{L}_{\mathrm{i}+1}-\mathrm{L}=\mathrm{f}\left(c_{i}\right)-\mathrm{L}
$$

O termo $T^{\prime}{ }_{i+1}$ refere-se somente à carga gerada pela deformação adicional. Dessa forma, é necessário considerar também a tração inicial $T_{o}$ :

$$
T_{i+1}=T_{i+1}^{\prime}+T_{o}
$$

\section{Exemplo de Aplicação}

Considera-se um cabo de comprimento inicial $L$ igual a 2 metros preso em duas extremidades, conforme mostra a Figura 4, estando o mesmo distribuído de um peso $p$ igual a $600 \mathrm{~N} / \mathrm{m}$, sendo que uma das extremidades está tracionada por uma carga $T_{0}$ igual a 10000 N. O cabo possui uma área da seção transversal 
$A$ igual a $1^{*} 10^{-4} \mathrm{~m}^{2}$ e um módulo de elasticidade $E$ igual a 100GPa.Tais valores são usualmente encontrados em algumas aplicações práticas relativas à engenharia estrutural e mecânica.

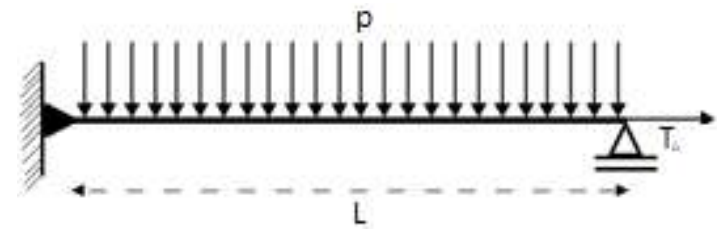

Figura 4- Cabo preso nas duas extremidades sujeito a cargas.

Realizam-se as iterações com base nas equações (28) a (32). Os resultados estão apresentados nas tabelas 1 e 2 .

Tabela 1- Iterações para a aproximação parabólica.

\begin{tabular}{cccc}
\hline Iteraçāo $(i)$ & $T_{i}(\mathrm{~N})$ & $\Delta \boldsymbol{L}_{i+1}(\mathrm{~m})$ & $\boldsymbol{L}_{i+1}(\mathrm{~m})$ \\
\hline 0 & 10000,00 & 0,00120 & 2,00120 \\
\hline 1 & 15996,76 & 0,00047 & 2,00047 \\
\hline 2 & 12344,20 & 0,00079 & 2,00079 \\
\hline 3 & 13936,15 & 0,00062 & 2,00062 \\
\hline 4 & 13088,48 & 0,00070 & 2,00070 \\
\hline 5 & 13501,35 & 0,00066 & 2,00066 \\
\hline 6 & 13290,55 & 0,00068 & 2,00068 \\
\hline 7 & 13395,73 & 0,00067 & 2,00067 \\
\hline 8 & 13342,63 & 0,00067 & 2,00067 \\
\hline
\end{tabular}

Tabela 2- Iterações para a aproximação hiperbólica.

\begin{tabular}{cccc}
\hline Iteração (i) & $\boldsymbol{T}_{i}(\mathrm{~N})$ & $\Delta \boldsymbol{L}_{i+1}(\mathrm{~m})$ & $\boldsymbol{L}_{i+1}(\mathrm{~m})$ \\
\hline 0 & 10000,00 & 0,00120 & 2,00120 \\
\hline 1 & 16001,08 & 0,00047 & 2,00047 \\
\hline 2 & 12343,60 & 0,00079 & 2,00079 \\
\hline 3 & 13938,39 & 0,00062 & 2,00062 \\
\hline 4 & 13088,63 & 0,00070 & 2,00070 \\
\hline 5 & 13502,74 & 0,00066 & 2,00066 \\
\hline 6 & 13291,17 & 0,00068 & 2,00068 \\
\hline 7 & 13396,79 & 0,00067 & 2,00067 \\
\hline 8 & 13343,44 & 0,00067 & 2,00067 \\
\hline
\end{tabular}

\section{Conclusões}

Da análise comparativa dos resultados obtidos na simulação dos modelos parabólico e hiperbólico percebe-se que a diferença nos resultados não é significativa, mostrando que para os níveis de deslocamento transversal usualmente obtidos nas aplicações de engenharia, em que a flecha máxima ébem inferior ao comprimento do vão, pode-se usar qualquer um dos dois modelos apresentados.
A conclusão mais importante, contudo, refere-se à necessidade da consideração do modelo constitutivonão linear. Verifica-se que houveram significativasalterações pela comparação dos valores de tração e deslocamentos obtidos na primeira iteração com os valores definitivos, alcançados na oitava iteração.

Por fim, uma vez que o esquema equaciona matematicamente problemas não lineares, a convergência pode não ser alcançada para situações em que o valor da pré-tração é reduzido com relação ao carregamento imposto, ou seja, os casos em que a tração no cabo é fortemente dependente do deslocamento. Em tais situações a não linearidade prepondera e a estabilidade do modelo deve ser garantida por artifícios matemáticos elaborados [9].

\section{Referências}

[1] LOEFFLER C. F., PRODONOFF V.Análise Dinâmica Elasto-Plástica de Torres Estaiadas,Anais do III Congresso Brasileiro de Energia, Rio de Janeiro, vol 5, pag 1867-1876, 1984.

[2] THOMAS JR. G. B., Cálculo, vol III, LTC Editora, Rio de Janeiro, 1974.

[3] BOYCE, W., DIPRIMA R.C. EquaçõesDiferenciais Elementares e Problemas de Valor de Contorno, LTCEditora, Rio de Janeiro, 2010.

[4] ODEN, T. J.; Mechanics of Elastic Structures. New York: McGraw-Hill, 1967.

[5]KREYSZIG E.; Matemática Superior para Engenharia, LTC Editora,Rio de Janeiro, 2009.

[6] COIMBRA, A. L.; Lições de Mecânica do Contínuo. São Paulo: Edgar Blucher, 1978.

[7] TIMOSHENKO, S. P.; GOODIER, J. N.; Theory of Elasticity. New York: McGraw-Hill, 1970.

[8] MALVERN L. E.; Introduction to the Mechanics of a Continuous Medium. New Jersey: Prentice-Hall, 1969.

[9] FERTIS D. G.; Nonlinear Mechanics, CRC Pres, London, 1998. 